\title{
MULTIPLE METASTASES GESTATIONAL CHORIOCARCINOMA - A CASE REPORT
}

\author{
doi: 10.2478/rojost-2018-0012
}

C. Mehedințu ${ }^{1,2}$, M.M. Cîrstoiv ${ }^{1}$, E. Brătilă ${ }^{1}$, M. Plotogea ${ }^{1,2}$, M. Antonovici ${ }^{1,2}$, A. Cazachevici ${ }^{2}$, C. Berceanu ${ }^{3}$

"Carol Davila" University of Medicine and Pharmacy, Bucharest, Romania

"Nicolae Malaxa" Clinical Hospital, Bucharest, Romania

${ }^{3}$ Craiova University of Medicine and Pharmacy, Craiova, Romania

Introduction. Gestational choriocarcinoma (GC) is a malignant condition that develops form trophoblastic cells originating from a previous pregnancy, eutopic or ectopic. Metastatic choriocarcinoma is often diagnosed in the absence of a primary uterine or ovarian tumor, presumably because the latter has undergone complete necrosis. GC rapidly metastasizes spreading to lungs, brain, bones, bone marrow, liver, and other organs, but responds well to chemotherapy.

Material and methods. We present the case of a 25 -year-old woman with critical evolution after being diagnosed with choriocarcinoma following a complete hydatidiform mole pregnancy. Even though the patient initially received proper treatment, due to poor follow-up compliance, the condition led to invasive metastases. When it spread to the musculoskeletal system and other organs, doctors were able to link symptoms to the choriocarcinoma diagnosis and chemotherapy was initiated. Unfortunately, the patient succumbed due to intracranial hemorrhage.

Discussions. GC is a highly and fast invasive malignancy, with rapidly growing tumors. It is often associated with hemorrhage, ischemic necrosis, and secondary inflammation. Often, by the time the tumor is discovered, $\mathrm{X}$-rays of the chest and bones already show metastatic lesions. Chemotherapy success depends on early detection and diagnosis of tumors after pregnancy.

Conclusions. Even though the initial diagnosis and treatment should have led to a favorable outcome, the patient's poor compliance was followed by rapid progression of the disease and, finally, exitus.

Keywords: gestational choriocarcinoma, metastases, chemotherapy 